Eur. Phys. J. C manuscript No.

(will be inserted by the editor)

\title{
A new model for spherically symmetric anisotropic compact star
}

\author{
S.K. Maurya $a^{a \sqrt{1}}$, Y.K. Gupta ${ }^{b \mid 2}$, Baiju \\ Dayanandane 3 , Saibal Ray \\ ${ }^{1}$ Department of Mathematical \& Physical Sciences, College of Arts \& Science, University of \\ Nizwa, Nizwa, Sultanate of Oman \\ ${ }^{2}$ Department of Mathematics, Raj Kumar Goel Institute of Technology, Ghaziabad, \\ U.P., India \\ ${ }^{3}$ Department of Mathematical \& Physical Sciences, College of Arts \& Science, University \\ of Nizwa, Nizwa, Sultanate of Oman \\ ${ }^{4}$ Department of Physics, Government College of Engineering \& Ceramic Technology, \\ Kolkata 700010, West Bengal, India \\ Received: date / Accepted: date
}

\begin{abstract}
In this article we obtain a new anisotropic solution for Einstein's field equation of embedding class one metric. The solution is representing the realistic objects such as $\operatorname{Her} X-1$ and $R X J 1856-37$. We perform detailed investigation of both objects by solving numerically the Einstein field equations under with anisotropic pressure. The physical features of the parameters depend on the anisotropic factor i.e. if anisotropy is zero everywhere inside the star then the density and pressures will become zero and metric turns out to be flat. We report our results and compare with the above mentioned two compact objects on a number of key aspects: the central density, the surface density onset and the critical scaling behavior, the effective mass and radius ratio, the anisotropization with isotropic initial conditions, adiabatic index and red shift. Along with this we have also made a comparison between the classical limit and theoretical model treatment of the compact objects. Finally we discuss the implications of our findings for the stability condition in relativistic compact star.
\end{abstract}

Keywords general relativity; metric functions; anisotropic factor; Compact stars

\section{Introduction}

Recent development in cosmological deep survey has clarified progressively the origin and distribution of matter and evolution of compact objects in the Universe. Some of their properties, such as masses, rotation frequencies, and emission of radiation are measurable, whereas measurements of important parameters which decide the nature of compact stars still represent an observational challenge. The properties that are not directly linked to observations, such as the internal composition or masses and radii, require the development of theoretical models. On

\footnotetext{
ae-mail: sunil@unizwa.edu.om

be-mail: kumar001947@gmail

ce-mail: baiju@unizwa.edu.om

de-mail: saibal@associates.iucaa.in
} 
the theoretical side, the mass and the radius are determined by solving the hydrostatic equilibrium equation which expresses the equilibrium between gravitational and pressure forces. In the framework of the general relativity the equilibrium of a spherical object is described by the Tolman-Oppenheimer-Volkoff (TOV) [1,2 equations and for completeness, the equation of state is required. Very recently other theoretical advances in modeling of densely neutral gravitating objects in strong gravitational fields has generated much interest in last couple of decades. This is because of its importance in describing relativistic astrophysical objects such as neutron stars, quark stars, hybrid proto-neutron stars, bare quark stars, etc.

The main theoretical routes have been used to study of stellar structure and evolution is that the interior of a star can be modeled as perfect fluid. The perfect fluid model necessarily requires the pressure in the interior of a star to be isotropic. Spacetime fueled by rotating anisotropic fluid has been used to model the interior of the star. One common source is a fluid with anisotropy in pressure. In particular at very high densities conventional celestial bodies are not composed purely of perfect fluids so that radial pressures are different from tangential pressures. The model of Bowers and Liang [3] is conceptually different form isotropic matter, but possess anisotropic matter in the study of general relativity. Mak and Harko [4, Sharma et al. [5] suggest that anisotropy is a sufficient condition in the study of dense nuclear matter with strange star. Some argument against the existence of anisotropy, could be verifiable through the existence of solid core or presence of type 3A fluid, were given by Kippenhahn and Weigert [6]. On the other hand, this can arise from different kind of phase transition and pion condensation as pointed out by Sokolov and Sawyer [7]. The structure of compact objects in general relativity will depend on several parameters, including fluid and magnetic stresses, entropy gradients, composition, heat flow and neutrino emission. However, we restrict our attention to the case of anisotropic perfect fluid with equilibrium composition.

The theoretical investigation of compact objects has been done by several workers by using both analytical and numerical methods. However, emphasize has been always given on the importance of local pressure anisotropy. This seems to be very reasonable to explain the matter distribution under a variety of circumstances. Also, this has been proved to be very useful to explore characteristics of relativistic compact objects [8, 9, 10,11,12,13, 14, 15]. In recent years, many exact solutions to the Einstein field equations have been generated by different approaches 16, 17 . 18, 19, 20, 21, 22. Therefore, the Einstein-Maxwell spacetime geometry for a compact object having local anisotropic effect has attracted considerable attention in various physical investigations. However, physical acceptability of the solution depends on the number of criteria which include fulfillment of various energy conditions of general relativity.

Under this background we would like to mention that in one of the earlier works Maurya et al. 23. have proposed an algorithm of charged anisotropic compact star while in the later works [24,25] they have given a new approach for finding out an anisotropic solution of Einstein's field equations by using the metric potentials function. The present work is a sequel of the work done by Maurya et al. [26, 27, in which the authors have obtained the charged compact star and the structure of relativistic electromagnetic mass model under the condition of class one metric. It would be desirable to do a systematic stability analysis of our model based on 
anisotropic spacetime. In this work, we check the mass-radius relation, stability and surface redshift of our models and found out their behavior is well behaved.

The present article is organized as follows: The Sect. 2 contains the spherical symmetric metric and the Einstein field equations. Also we find the metric function $\lambda$ in terms of metric function $\nu$ by applying the class one condition. In Sect. 3, we obtain interior structure of the anisotropic star under the class one condition. The values of arbitrary constants and total mass of the compact star of radius $R$ are obtained by using the boundary conditions in Sect. 4 . In Sect. 5 , we discuss about several required physical conditions for anisotropic models along with the stability analysis which is vital one. The last Sect. 6 contains some concluding remarks on the anisotropic models.

\section{Line element for class one metric and Einstein's field equations}

We consider the static spherically symmetric metric for describing the spacetime of compact stellar configuration as

$$
d s^{2}=-e^{\lambda} d r^{2}-r^{2}\left(d \theta^{2}+\sin ^{2} \theta d \phi^{2}\right)+e^{\nu} d t^{2} .
$$

The energy momentum tensor of interior matter for a strange star may be expressed in the following standard form

$$
T_{i j}=\operatorname{diag}\left(\rho,-p_{r},-p_{t},-p_{t}\right) \text {, }
$$

where $\rho, p_{r}$ and $p_{t}$ are corresponding to the energy density, radial and tangential pressures respectively of matter distribution.

The Einstein field equations can be written as

$$
R_{i j}-\frac{1}{2} R g_{i j}=-8 \pi T_{i j}
$$

Here $G=c=1$ under the geometrized relativistic units.

In view of the metric (1), Eq. (3) yields the following differential equations [28]

$$
\begin{gathered}
p_{r}=\frac{e^{-\lambda}}{8 \pi}\left[\frac{v^{\prime}}{r}-\frac{\left(e^{\lambda}-1\right)}{r^{2}}\right], \\
p_{t}=\frac{e^{-\lambda}}{8 \pi}\left[\frac{v^{\prime \prime}}{2}-\frac{\lambda^{\prime} v^{\prime}}{4}+\frac{v^{\prime 2}}{4}+\frac{v^{\prime}-\lambda^{\prime}}{2 r}\right], \\
\rho=\frac{e^{-\lambda}}{8 \pi}\left[\frac{\lambda^{\prime}}{r}+\frac{\left(e^{\lambda}-1\right)}{r^{2}}\right] .
\end{gathered}
$$

The metric (1) may represent spacetime of the embedding class one, if it satisfies the condition of Karmarker [29. This condition gives the following relation between the metric potentials $\nu$ and $\lambda$ as 24 .

$$
e^{\lambda(r)}=1+C \nu^{\prime 2} e^{\nu} .
$$




\section{New anisotropic models for compact star}

To find interior solution of the anisotropic compact star in class one, we consider the pressure isotropy condition as given through the expression of the anisotropic factor as follows

$$
\frac{e^{-\lambda}}{8 \pi}\left[\frac{v^{\prime \prime}}{2}-\frac{\lambda^{\prime} v^{\prime}}{4}+\frac{v^{\prime 2}}{4}-\frac{v^{\prime}+\lambda^{\prime}}{2 r}+\frac{e^{\lambda}-1}{r^{2}}\right]=\left(p_{t}-p_{r}\right)=\Delta .
$$

For finding out the non-zero expression for the anisotropic factor, we assume the metric potential in the form

$$
e^{\nu}=B e^{2 A r^{2}}
$$

where $A$ and $B$ are positive constants.

Hence from Eqs. (7) and (9), we get

$$
e^{\lambda}=\left[1+D A r^{2} e^{2 A r^{2}}\right]
$$

where

$$
D=16 A B C .
$$

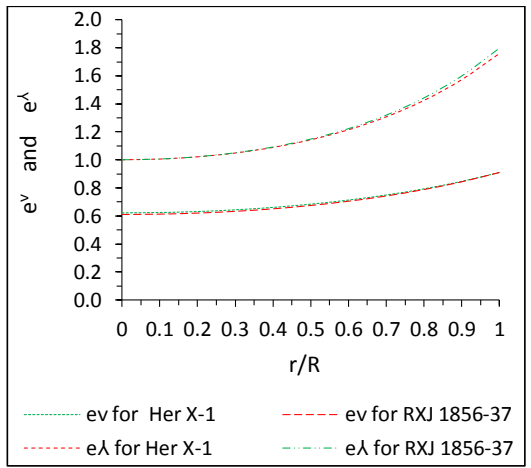

Fig. 1 Variation of metric potentials $e^{\nu}$ and $e^{\lambda}$ with the radial coordinate $r / R$ for $\operatorname{Her} X-1$ and $R X J 1856-37$

From Eqs. (9) and (10), we observe that $e^{\lambda(0)}=1$ and $e^{\nu(0)}=B$ at the centre $r=0$. This shows that metric potentials are singularity free and positive at the centre. Also both are monotonically increasing function which shows that these metric potential are physically valid [30. These features can be observed from Fig. (1).

By plugging Eqs. (9) and (10) into Eq. (8), we get

$$
\Delta=\frac{A^{2} r^{2}}{8 \pi}\left[\frac{-2+D e^{2 A r^{2}}}{1+D A r^{2} e^{2 A r^{2}}}\right]^{2} .
$$

We note from Fig. (2) that the anisotropy $\Delta$ is zero at the centre $r=0$ and is monotonically increasing with increase of $r$. Also from Eq. (12), we observe that 


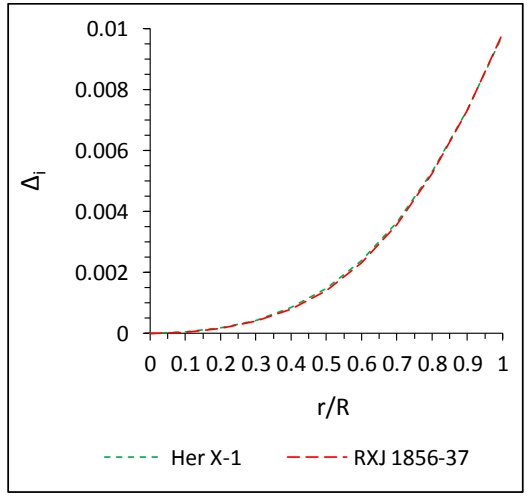

Fig. 2 Variation of anisotropic factor $\Delta$ with the radial coordinate $r / R$ for $H e r X-1$ and RXJ $1856-37$

the anisotropic factor $\Delta$ vanishes everywhere inside the compact star if and only if $A=0$.

Eqs. (4), (5) and (6) give expressions for the radial pressure $p_{r}$, the tangential pressure $p_{t}$ and the energy density $\rho$ as

$$
\begin{gathered}
p_{r}=\frac{A}{8 \pi}\left[\frac{4-D e^{2 A r^{2}}}{1+D A r^{2} e^{2 A r^{2}}}\right], \\
p_{t}=\frac{A}{8 \pi}\left[\frac{4-D e^{2 A r^{2}}+4 A r^{2}}{\left(1+D A r^{2} e^{2 A r^{2}}\right)^{2}}\right], \\
\rho=\frac{A}{8 \pi}\left[\frac{D e^{2 A r^{2}}\left(3+4 A r^{2}+A r^{2} D e^{2 A r^{2}}\right)}{\left(1+D A r^{2} e^{2 A r^{2}}\right)^{2}}\right] .
\end{gathered}
$$

The radial and tangential pressures at centre $r=0$ can be given by $p_{r}=$ $A(4-D) / 8 \pi$ and $p_{t}=A(4-D) / 8 \pi$. Since $A$ and $D$ are positive and pressure should be positive at the centre, therefore this implies that $D<4$. In a similar way, we can find out the density at the centre $\mathrm{r}=0$ as $\rho_{0}=(3 A D / 8 \pi)$. Since the density should be positive at the centre, then $D$ is positive due to positivity of $A$. As $D, A, B$ all are positive, therefore this implies that $C$ is also a positive quantity.

We suppose that the radial and tangential pressures of the star are related to the matter density by a parameters $\omega_{r}$ as $p_{r}=\omega_{r} \rho$ and $p_{t}=\omega_{t} \rho$.

Then the expressions for the parameters $\omega_{r}$ and $\omega_{t}$ are given by

$$
\begin{aligned}
& \omega_{r}=\frac{\left(4-D e^{2 A r^{2}}\right)\left(1+D A r^{2} e^{2 A r^{2}}\right)}{D e^{A r^{2}}\left[3+4 A r^{2}+A r^{2} D e^{2 A r^{2}}\right]}, \\
& \omega_{t}=\frac{4\left(1+A r^{2}\right)-D e^{2 A r^{2}}}{D e^{2 A r^{2}}\left[3+4 A r^{2}+A r^{2} D e^{2 A r^{2}}\right]} .
\end{aligned}
$$

Form Fig. (5) it is clear that the ratio $\omega_{r}=p_{r} / \rho$ and $\omega_{t}=p_{t} / \rho$ are less than 1. This implies that density is dominating over the pressures throughout inside the star. However this also implies that the underlying fluid distribution is non-exotic in nature 39 . 

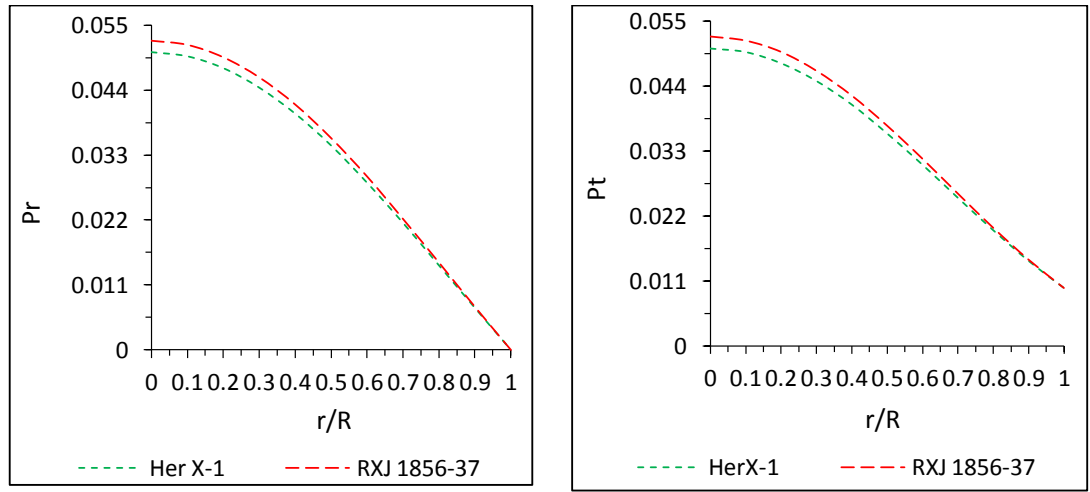

Fig. 3 Variation of radial pressure (left panel) and transverse pressure (right panel) with respect to the fractional radius $r / R$ for $\mathrm{Her} X-1$ and $R X J 1856-37$

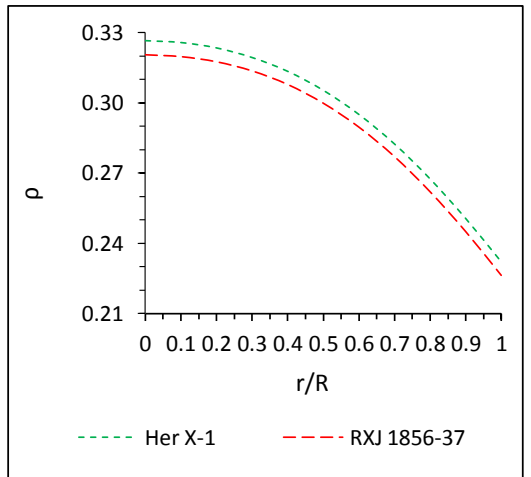

Fig. 4 Variation of energy density $(\rho)$ with respect to the fractional radius $r / R$ for $\operatorname{Her} X-1$ and $R X J 1856-37$

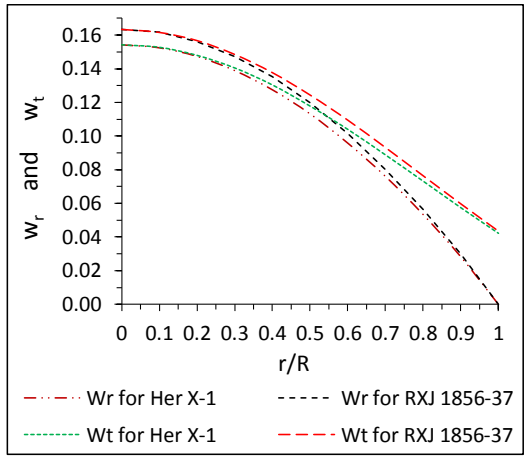

Fig. 5 Variation of parameters $\omega_{r}$ and $\omega_{t}$ with the radial coordinate $r / R$ for $H e r X-1$ and $R X J 1856-37$ 
4 Matching condition

For any physically acceptable anisotropic solution, the following boundary conditions must be satisfied:

(i) At the surface of the compact star, the interior of metric (1) for anisotropic matter distribution match with the exterior of Schwarzschild solution [31, which is given by the metric

$$
d s^{2}=\left(1-\frac{2 M}{r}\right) d t^{2}-r^{2}\left(d \theta^{2}+\sin ^{2} \theta d \phi^{2}\right)-\left(1-\frac{2 M}{r}\right)^{-1} d r^{2},
$$

where $M$ is a constant representing the total mass of the compact star at $r=R$.

(ii) The radial pressure $p_{r}$ must be finite and positive at the centre $r=0$ and it must vanish at the surface $r=R$ of the star 32 . The condition $p_{r}(R)=0$ gives

$$
D=16 A B C=4 e^{-2 A R^{2}}
$$

This readily yields the radius $R$ of the compact star as

$$
R=\sqrt{\frac{1}{2 A} \ln \left[\frac{1}{4 A B C}\right]} .
$$

Using the continuity of metric coefficients $e^{\nu}, e^{\lambda}$ and $\frac{\partial g_{t t}}{\partial r}$ across the boundary of the star gives the following equations

$$
\begin{gathered}
B e^{2 A R^{2}}=1-\frac{2 M}{R} \\
1+D A R^{2} e^{2 A R^{2}}=\left[1-\frac{2 M}{R}\right]^{-1} \\
4 B A R^{2} e^{2 A R^{2}}=\frac{2 M}{R}
\end{gathered}
$$

These following equations with Eq. (19) gives the value of unknowns M, B and $\mathrm{C}$ as follows:

$$
\begin{gathered}
M=\frac{R}{2}\left[\frac{4 A R^{2}}{1+4 A R^{2}}\right] \\
B=\frac{e^{-2 A R^{2}}}{1+A R^{2}} \\
C=\frac{1+A R^{2}}{4 A} .
\end{gathered}
$$

On the other hand, the value of the constant $A$ can be determined by assuming the density at the surface of the star i.e. $\rho_{s}$ at $r=R$. 


\section{Physical Features of the anisotropic models}

\subsection{Sound speed}

The speed of sound should monotonically decrease thought out from the centre to the boundary of the star and it must be within the range, i.e. $0 \leq V_{i}=\sqrt{d p_{i} / d \rho}<$ 1. It is argued by Canuto 33 that the sound speed should decrease outwards for the EOS with an ultra-high distribution of matter. Form Fig. (6), it is clear that the speed of sound is monotonically decreasing outwards.

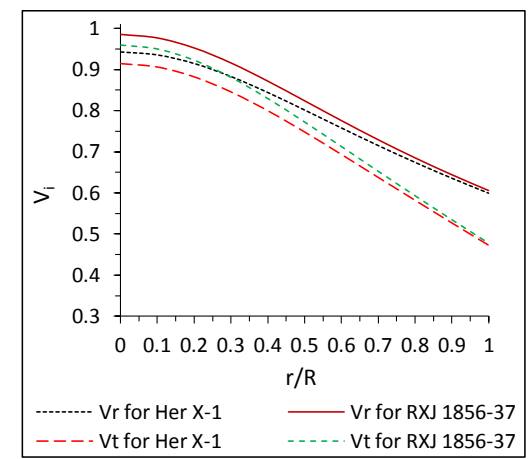

Fig. 6 Variation of sound speed with the radial coordinate $r / R$ for the $\operatorname{Her} X-1$ and $R X J 1856-37$

\subsection{Energy conditions}

The anisotropic fluid must satisfy the following energy conditions: Null energy condition (NEC), Weak energy condition (WEC) and Strong energy condition (SEC). Therefore, the following inequalities should hold simultaneously at each points inside the compact star corresponding to the above conditions:

$$
N E C: \rho \geq 0
$$

$W E C_{r}: \rho-p_{r} \geq 0$,

$W E C_{t}: \rho-p_{t} \geq 0$

$$
S E C: \rho-p_{r}-2 p_{t} \geq 0 \text {. }
$$



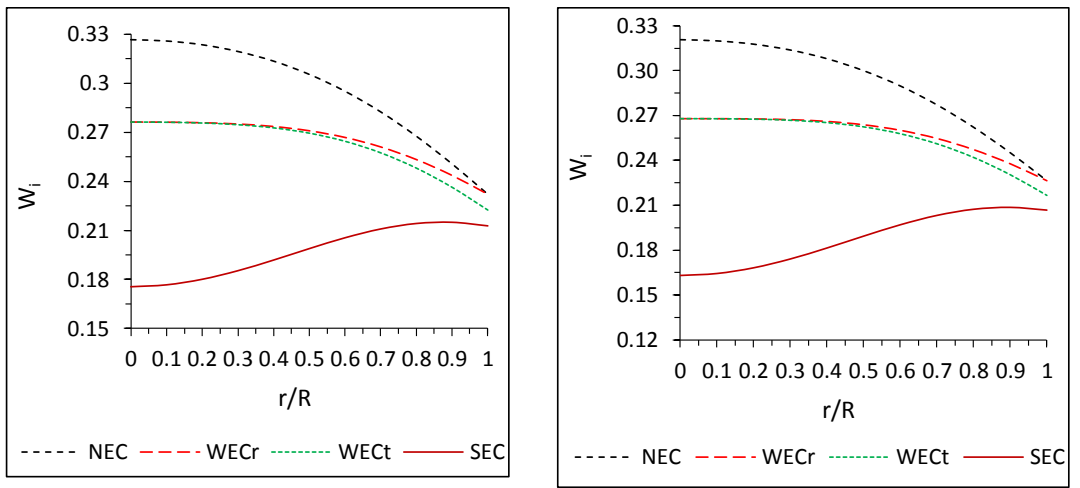

Fig. 7 Variation of energy conditions with the radial coordinate $r / R$ for the Her $X-1$ (left panel) and $R X J 1856-37$ (right panel)

\subsection{Tolman-Oppenheimer-Volkoff equation (TOV)}

The generalized TOV equation for the anisotropic fluid distribution is given by 1 , 2

$$
\frac{M_{G}\left(\rho+p_{r}\right)}{r^{2}} e^{\frac{\lambda-\nu}{2}}+\frac{d p_{r}}{d r}+\frac{2}{r}\left(p_{r}-p_{t}\right)=0 .
$$

We can write the above TOV equations as follows:

$$
-\frac{1}{2} \nu^{\prime}\left(\rho+p_{r}\right)-\frac{d p_{r}}{d r}+\frac{2}{r}\left(p_{t}-p_{r}\right)=0
$$

where $M_{G}$ is the effective gravitational mass and can be given by

$$
M_{G}(r)=\frac{1}{2} r^{2} e^{\frac{\nu-\lambda}{2}} \nu^{\prime}
$$

Eq. (32) describes the equilibrium condition for an anisotropic fluid distribution subject to the gravitational $\left(F_{g}\right)$, the hydrostatic $\left(F_{h}\right)$ and the anisotropic stress $\left(F_{a}\right)$ so that

$$
F_{g}+F_{h}+F_{a}=0,
$$

where its components can be defined as

$$
\begin{gathered}
F_{g}=-\frac{1}{2} \nu^{\prime}\left(\rho+p_{r}\right), \\
F_{h}=-\frac{d p_{r}}{d r} \\
F_{a}=\frac{2}{r}\left(p_{t}-p_{r}\right) .
\end{gathered}
$$

The explicit form of the above forces can be expressed as

$$
F_{g}=-\frac{A^{2} r}{4 \pi}\left[\frac{4+2 D e^{2 A r^{2}}\left(1+4 A r^{2}\right)}{\left(1+D A r^{2} e^{2 A r^{2}}\right)^{2}}\right],
$$




$$
\begin{gathered}
F_{h}=-\frac{A^{2} r}{4 \pi}\left[\frac{D e^{2 A r^{2}}\left(-6+2 D e^{2 A r^{2}}-8 A r^{2}\right)}{\left(1+D A r^{2} e^{2 A r^{2}}\right)^{2}}\right] \\
F_{a}=\frac{A^{2} r}{4 \pi}\left[\frac{-2+D e^{2 A r^{2}}}{\left(1+D A r^{2} e^{2 A r^{2}}\right)}\right]^{2}
\end{gathered}
$$
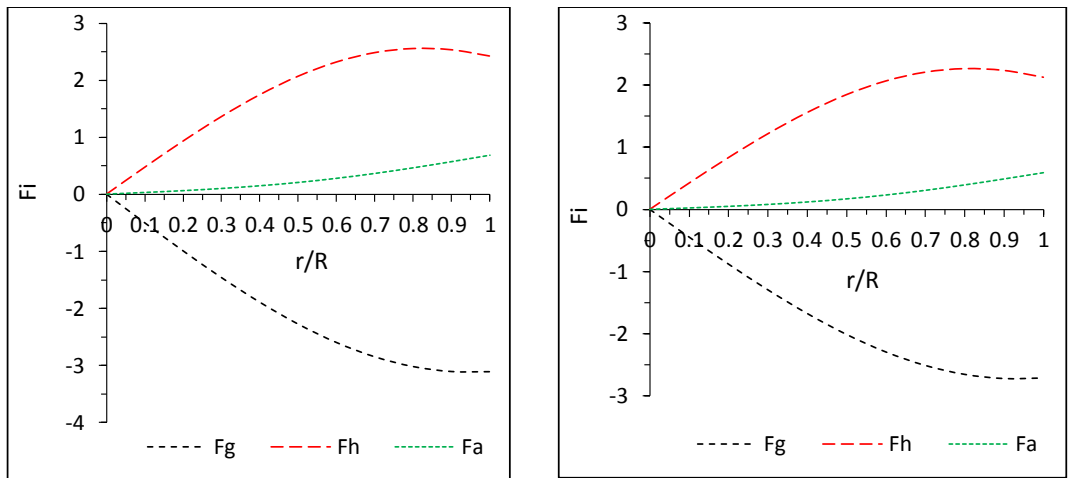

Fig. 8 Variation of different forces with the radial coordinate $r / R$ for $H e r X-1$ (left panel) and $R X J 1856-37$ (right panel)

\subsection{Stability of the models}

\subsubsection{Herrera cracking concept}

We know that for physically acceptable anisotropic models, the radial and transverse speed of sound should lies between 0 and 1 i.e. $0 \leq V_{r}<1$ and $0 \leq V_{t}<1$. We observe from this inequality that parameters also should satisfy the inequality $0 \leq V_{r}^{2}<1$ and $0 \leq V_{t}^{2}<1$. Now we define the expression for the square of velocity of sound as

$$
\begin{gathered}
V_{r}^{2}=\frac{d p_{r}}{d \rho}=\left[\frac{\left(6-D e^{2 A r^{2}}+8 A r^{2}\right)\left(1+D A r^{2} e^{2 A r^{2}}\right)}{D^{2} A r^{2} e^{4 A r^{2}}-2\left(5+4 A r^{2}\right)+D e^{2 A r^{2}}\left(5+6 A r^{2}+8 A^{2} r^{4}\right)}\right] . \quad \text { (41) } \\
V_{t}^{2}=\frac{d p_{t}}{d \rho}=\left[\frac{2\left[D e^{2 A r^{2}}\left(5+10 A r^{2}+8 A^{2} r^{4}\right)-2-D^{2} e^{4 A r^{2}}\left(1+A r^{2}\right)\right]}{D e^{2 A r^{2}}\left[D^{2} A r^{2} e^{4 A r^{2}}-2\left(5+4 A r^{2}\right)+D e^{2 A R^{2}}\left(5+6 A r^{2}+8 A^{2} r^{4}\right)\right.}\right] .
\end{gathered}
$$

From Fig. (9), we conclude that square of radial and transverse speeds of sound are lies within the range everywhere inside the stars. Therefore, $0 \leq\left|V_{t}^{2}-V_{r}^{2}\right|<1$. In order to examine the stability of local anisotropic fluid distribution, we follow the cracking concept of Herrera and Aberu et al. 34,35] which states that the 


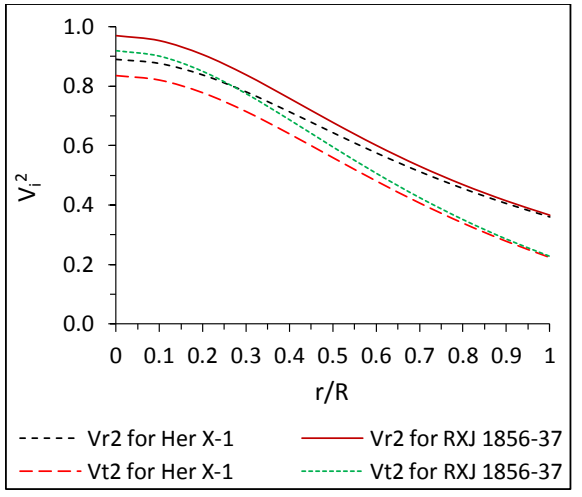

Fig. 9 Variation of square of radial speed of sound and transverse speed of sound with radial coordinate $r / R$ for Her $X-1$ and $R X J 1856-37$

region is potentially stable where the radial speed of sound is greater than the transverse speed of sound. This implies that there is no change in sign $V_{r}^{2}-V_{t}^{2}$ and $V_{t}^{2}-V_{r}^{2}$.

So calculate the difference between the radial and transverse speed of sound

$V_{t}^{2}-V_{r}^{2}=\left[\frac{\left(2-D e^{2 A r^{2}}\right)\left[2+D^{2} A r^{2} e^{4 A R^{2}}-D e^{2 A r^{2}}\left(1+6 A r^{2}+8 A^{2} r^{4}\right)\right]}{D e^{2 A r^{2}}\left[D^{2} A r^{2} e^{4 A r^{2}}-2\left(5+4 A r^{2}\right)+D e^{2 A r^{2}}\left(5+6 A r^{2}+8 A^{2} r^{4}\right)\right.}\right]$.
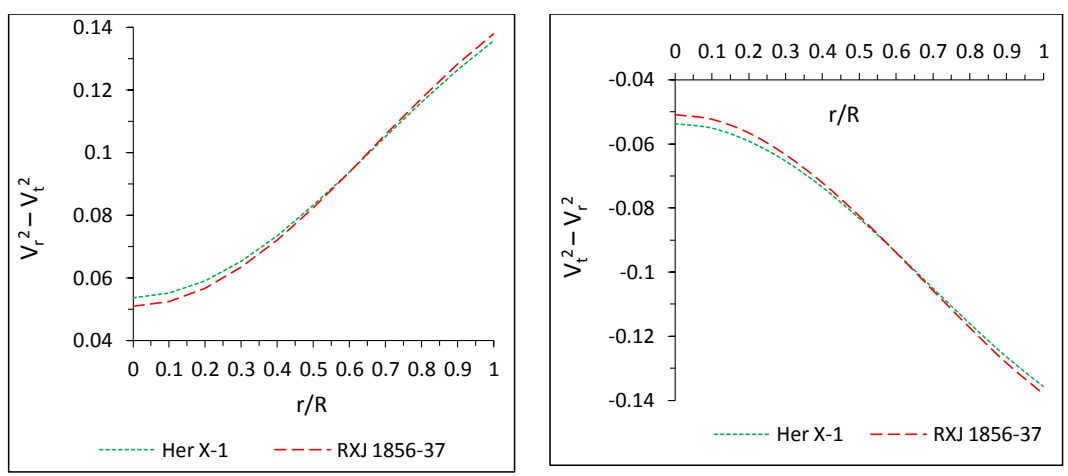

Fig. 10 Variation of difference between square of radial speed and transverse speed of sound with radial coordinate $r / R$ for $\operatorname{Her} X-1$ and $R X J 1856-37$

We note from Fig. (10) that radial speed of sound is always greater than transverse speed of sound and also $0 \leq\left|V_{t}^{2}-V_{r}^{2}\right|<1$ everywhere inside the star. These features represent that the proposed physical models are stable. 


\subsubsection{Adiabatic index}

In order to determine an equilibrium configuration, the matter must be stable against the collapse of local regions. This also requires Le Chatelier's principle (known as the local or microscopic stability condition) that the radial pressure $p_{r}$ must be a monotonically non-decreasing function of $\rho$ such that $\frac{d p_{r}}{d \rho}>$ 0 [36. Heintzmann and Hillebrandt 37 also proposed that a neutron star with an anisotropic equation of state is stable for $\gamma\left(=\frac{p_{r}+\rho}{p_{r}} \frac{d p_{r}}{d \rho}\right)>4 / 3$. From Fig. (11), it is clear that the adiabatic index $(\gamma)$ is more than $4 / 3$ everywhere inside the star.

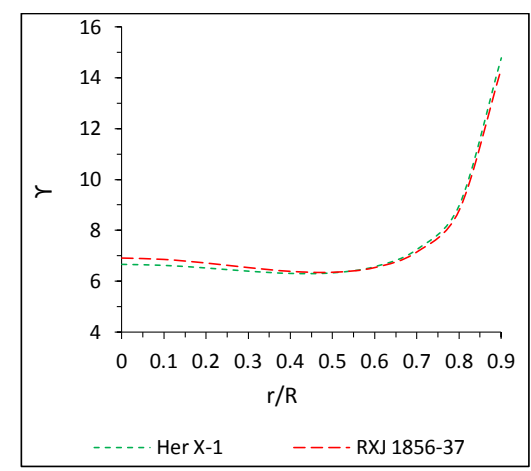

Fig. 11 Variation of adiabatic index $(\gamma)$ with radial coordinate $\mathrm{r} / \mathrm{R}$ for $\mathrm{Her} X-1$ and $R X J 1856-37$

\subsection{Effective mass-radius ratio}

This section contains the maximum allowable mass-radius ratio for above proposed anisotropic fluid models. As Buchdahl 38 has already discussed that the maximum limit of mass-radius ratio for static spherically symmetric perfect fluid star should satisfy the following upper bound $2 M / R<8 / 9$. Also Mak and Harko 4 have given the generalized expression for the same mass-radius ratio.

The effective mass of the anisotropic compact star is defined as

$$
M_{e f f}=4 \pi \int_{0}^{R} \rho r^{2} d r=\frac{1}{2} R\left[1-e^{-\lambda(R)}\right]=\frac{R}{2}\left[\frac{D A R^{2} e^{2 A R^{2}}}{1+D A R^{2} e^{2 A R^{2}}}\right]=\frac{R}{2}\left[\frac{4 A R^{2}}{1+4 A R^{2}}\right] .
$$

However the compactness $u$ of the star can be expressed as

$$
u=\frac{M_{e f f}}{R}=\frac{1}{2}\left[\frac{4 A R^{2}}{1+A R^{2}}\right] .
$$


Table-1: Values of the model parameters Mass $\left(M_{\odot}\right)$, Radius $(R), D, A, B$ and $C$ for different compact stars

\begin{tabular}{lrrrrrrr}
\hline $\begin{array}{l}\text { Compact star } \\
\text { candidates }\end{array}$ & $\frac{M}{R}$ & $M\left(M_{\odot}\right)$ & $R(\mathrm{~km})$ & $D$ & $\begin{array}{r}A \\
\left(\mathrm{~km}^{-2}\right)\end{array}$ & $\begin{array}{r}B \\
\left(\mathrm{~km}^{2} / \mathrm{sec}^{2}\right)\end{array}$ & $\begin{array}{r}\mathrm{C} \\
\left(\mathrm{sec}^{2}\right)\end{array}$ \\
\hline Her. X-1 & 0.216 & 0.9825 & 6.7 & 2.7344 & $4.2371 \times 10^{-13}$ & 0.6231 & $6.4733 \times 10^{11}$ \\
RXJ 1856-37 & 0.222 & 0.9041 & 6.0 & 2.6834 & $5.5443 \times 10^{-13}$ & 0.6108 & $4.9528 \times 10^{11}$ \\
\hline
\end{tabular}

Table-2: The energy densities, the central pressure and $A R^{2}$ for different compact star candidates for the above parameter values of Table-1

\begin{tabular}{|c|c|c|c|c|}
\hline $\begin{array}{l}\text { Compact star } \\
\text { candidates }\end{array}$ & $\begin{array}{r}\text { Central Density } \\
\mathrm{gm} / \mathrm{cm}^{3}\end{array}$ & $\begin{array}{r}\text { Surface density } \\
\mathrm{gm} / \mathrm{cm}^{3}\end{array}$ & $\begin{array}{r}\text { Central pressure } \\
\text { dyne } / \mathrm{cm}^{2}\end{array}$ & $A R^{2}$ \\
\hline Her. X-1 & $1.8664 \times 10^{15}$ & $1.3273 \times 10^{15}$ & $2.5923 \times 10^{35}$ & 0.1902 \\
\hline RXJ 1856-37 & $2.3968 \times 10^{15}$ & $1.6924 \times 10^{15}$ & $3.5282 \times 10^{35}$ & 0.1996 \\
\hline
\end{tabular}

\subsection{Surface redshift}

The surface redshift $\left(Z_{s}\right)$ corresponding to the above compactness $(u)$ is given by the expression

$$
Z_{s}=\frac{1-[1-2 u]^{1 / 2}}{[1-2 u]^{1 / 2}}=\sqrt{1+4 A R^{2}}-1 .
$$

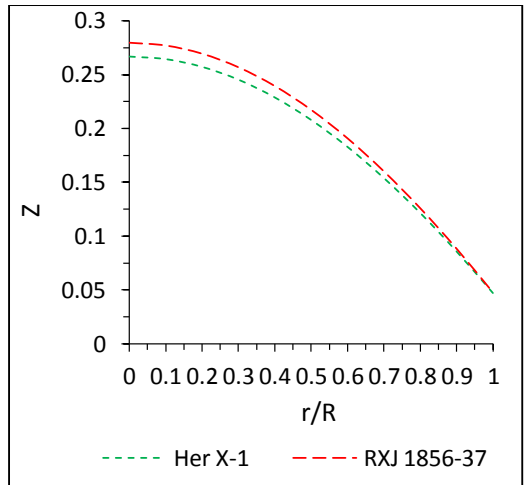

Fig. 12 Variation of the redshift $(Z)$ with the radial coordinate $r / R$ for $\operatorname{Her} X-1$ and $R X J 1856-37$

\section{Conclusions}

In the present article, we have obtained new anisotropic compact star models of embedding class one metric. Our models satisfy all the physical reality conditions. Some of the special features of the present model are as follows:

(1) We used the boundary conditions by joining the Schwarzschild metric with class one metric at the boundary of the star $r=R$. Subsequently we obtained the 
arbitrary constants $A, B, C$ along with the total mass of the compact star and the corresponding numerical values are provided in Table-1. All these values are matching with the observed data of the real compact stars.

(2) The metric potentials are free from any singularity at the centre, positive and finite inside the star (Fig. 1). Also the $\rho, p_{r}$ and $p_{t}$ are positive, finite and monotonically decreasing away from the centre. However, the parameters $\omega_{r}$ and $\omega_{t}$ are within the range i.e. lies between 0 and 1 (Fig. 5).

(3) The model is in static equilibrium. We observe from Fig. (8) that the gravitational force $\left(F_{g}\right)$ is dominating over the hydrostatic force $\left(F_{h}\right)$ and is counter balanced by the joint action of the hydrostatic force and the anisotropic stress.

(4) The model has density of the order $10^{15} \mathrm{gm} / \mathrm{cm}^{3}$. The corresponding values for Her $X-1$ and $R X J 1856-37$ are as follows:

(i) at the centre: $\rho_{0}=1.8664 \times 10^{15} \mathrm{gm} / \mathrm{cm}^{3}$ and $\rho_{0}=2.3968 \times 10^{15} \mathrm{gm} / \mathrm{cm}^{3}$,

(ii) at the surface: $\rho_{0}=1.3273 \times 10^{15} \mathrm{gm} / \mathrm{cm}^{3}$ and $\rho_{0}=1.6924 \times 10^{15} \mathrm{gm} / \mathrm{cm}^{3}$ (Table2).

This density profile shows that our models may represent a realistic anisotropic objects.

(5) The redshift is monotonically decreasing and attains its maximum value at the centre of the compact star. The numerical values corresponding to the Her $X-1$ and $R X J 1856-37$ are: (i) at the centre: $Z_{0}=0.2669$ and $Z_{0}=0.2796$, (ii) at the surface: $Z_{s}=0.0474$ and $Z_{s}=0.0480$.

As a final comment, an interesting and puzzling point about the anisotropic compact model is that its stability depends on the unavoidable anisotropic pressure and the TOV equations used to place constraint on the anisotropic parameters. It would be interesting to propose a richer model in which consideration of the pressure anisotropy on the compact relativistic objects could lead to a more realistic model of the anisotropization mechanism as regards to the compact relativistic objects.

\section{Acknowledgment}

The authors SKM and BD acknowledge continuous support and encouragement from the administration of University of Nizwa. SR wishes to thank the authorities of the Inter-University Centre for Astronomy and Astrophysics, Pune, India for providing him Visiting Associateship. We all are thankful to the anonymous referee for raising several pertinent issues which have helped us to improve the manuscript substantially.

\section{References}

1. R.C. Tolman, Phys. Rev. 55, 364 (1939)

2. J.R. Oppenheimer, G.M. Volkoff, Phys. Rev. 55, 374 (1939)

3. R.L. Bowers, E.P.T. Liang, Astrophys. J. 188, 657 (1974)

4. M.K. Mak, T. Harko, Proc. R. Soc. A 459, 393 (2003)

5. R. Sharma, S. Mukherjee, S. D. Maharaj, Gen. Relativ. Gravit. 33, 999 (2001)

6. R. Kippenhahm, A. Weigert, Stellar Structure and Evolution, Springer, Berlin (1990) 
7. A.I. Sokolov, J. Exp. Theor. Phys. 79, 1137 (1980)

8. L. Herrera, N. O. Santos, Phys. Rep. 286, 53 (1997)

9. L. Herrera, A. Di Prisco, J. Martin, J. Ospino, N. O. Santos, O. Troconis, Phys. Rev. D 69, 084026 (2004)

10. C. Cattoen, T. Faber, M. Visser, Class. Quantum Gravit. 22, 4189 (2005)

11. De Benedictis, D. Horvat, S. Ilijic, S. Kloster, K. Viswanathan, Class. Quantum Gravit. 23, 2303 (2006)

12. G. Bohmer, T. Harko, Classical Quantum Gravity 23, 6479 (2006)

13. W. Barreto, B. Rodriguez, L. Rosales, O. Serrano, Gen. Relativ. Gravit. 39, $23(2006)$

14. M. Esculpi, M. Malaver, E. Aloma, Gen. Relativ. Gravit. 39, 633 (2007)

15. G. Khadekar, S. Tade, Astrophys. Space Sci. 310, 41 (2007)

16. G. Bohmer, T. Harko, Mon. Not. R. Astron. Soc. 37, 393 (2007)

17. S. Karmakar, S. Mukherjee, R. Sharma, S. Maharaj, Pramana - J. phy. 68, 881 (2007)

18. H. Abreu, H. Hernandez, L. A. Nunez, Classical Quantum Gravity 24, 4631 (2007)

19. L. Herrera, A. Di Prisco, J. Ospino, E. Fuenmayor, J. Math. Phys. 42, 2129 (2001)

20. S.K. Maurya, Y.K. Gupta, Astrophys. Space Sci. 344, 243 (2013)

21. P.H. Nguyen, M. Lingam, Mon. N. Royal. Ast. Soc. 436, 2014 (2013)

22. M. Malaver, American Journal of Astronomy and Astrophysics 1, 41 (2013)

23. S.K. Maurya, Y.K. Gupta, S. Ray, arXiv: 1502.01915 [gr-qc] (2015)

24. S.K. Maurya, Y.K. Gupta, S. Ray, B. Dayanandan, Eur. Phys. J. C 75, 225 (2015)

25. S.K. Maurya, Y.K. Gupta, B. Dayanandan, M.K. Jasim, A. Al-Jamel, arXiv:1511.01625 [gr-qc] (2015)

26. S.K. Maurya, Y.K. Gupta, S. Ray, S. Roy Chowdhury, Eur. Phys. J. C 75, 389 (2015)

27. S.K. Maurya, Y.K. Gupta, S. Ray, V. Chatterjee, arXiv:1507.01862 [gr-qc] (2015)

28. D.D. Dionysiou, Astrophys. Space Sci. 85, 331 (1982)

29. K.R. Karmarkar, Proc. Ind. Acad. Sci. A 27, 56 (1948)

30. K. Lake, Phys. Rev. D 67, 104015 (2003)

31. K. Schwarzschild, Sitz. Deut. Akad. Wiss. Math.-Phys. Berlin 24, 424 (1916)

32. C. W. Misner, D.H. Sharp, Phys. Rev. B 136, 571 (1964)

33. V. Canuto, Solvay Conf. on Astrophysics and Gravitation, Brussels (1973)

34. L. Herrera: Phys. Lett. A 165, 206 (1992)

35. H. Abreu, H. Hernandez, L.A. Nunez: Class. Quantum Gravit. 24, 4631 (2007)

36. S.S. Bayin, Phys. Rev. D 261262 (1982)

37. H. Heintzmann, W. Hillebrandt, Astron. Astrophys. 38, 51 (1975)

38. H.A. Buchdahl, Phys. Rev. 116, 1027 (1959)

39. F. Rahaman, S.A.K. Jafry, K. Chakraborty, Phys. Rev. D 82, 104055 (2010) 